\title{
Accuracy of cone-beam computed tomography for the evaluation of mandible invasion by oral squamous cell carcinoma
}

\section{Zezheng Wang}

Nanjing Stomatological Hospital

\section{Shuang Zhang}

Nanjing Stomatological Hospital

\section{Yumei Pu}

Nanjing Stomatological Hospital

\section{Yuxin Wang}

Nanjing Stomatological Hospital

\section{Zitong Lin}

Nanjing Stomatological Hospital

\section{Zhiyong Wang ( $\nabla$ zywangkq@nju.edu.cn )}

Department of Oral and Maxillofacial Surgery, Nanjing Stomatological Hospital https://orcid.org/00000002-4065-0820

\section{Research article}

Keywords: CBCT, mandibular invasion, oral cancer, cancer, preoperative assessment

Posted Date: March 9th, 2020

DOI: https://doi.org/10.21203/rs.3.rs-16437/v1

License: (c) (i) This work is licensed under a Creative Commons Attribution 4.0 International License.

Read Full License

Version of Record: A version of this preprint was published at BMC Oral Health on May 1st, 2021. See the published version at https://doi.org/10.1186/s12903-021-01567-3. 


\section{Abstract}

Purpose: Surgery for oral cancer with mandibular invasion requires an accurate preoperative assessment. Although cone-beam computed tomography (CBCT), which yields high spatial resolution, is used widely for mandibular examinations, evidence supporting its accuracy for the evaluation of malignant mandibular invasion is insufficient. Therefore, this study aimed to explore the accuracy of CBCT for the preoperative assessment of mandibular invasion.

Methods: Thirty mandibular specimens acquired via mandibulectomy for oral squamous cell carcinoma (OSCC) treatment were collected. The excised samples were marked and subjected to CBCT imaging. Hematoxylin-eosin staining was used for histopathological assessment. Mandibular invasion was estimated based on CBCT and pathologic findings. Invasive borders were delineated via histopathological examination and CBCT and merged to compare the extent of invasion.

Results: Although CBCT predicted the excised mandible invasion with $100 \%$ accuracy, it predicted nerve invasion with only $69.2 \%$ accuracy. The bone samples exhibited significant shrinkage (8.9\%) due to tissue processing. This bone mass shrinkage led to a tendency of CBCT to underestimate the extent of invasion, with a mean difference of $2.97 \mathrm{~mm}$.

Conclusion: CBCT can reliably assess mandibular invasion, but is much less accurate for the estimation of nerve invasion. Given the potential for underestimation of the extent of mandibular invasion, CBCT would be better used to determine the extent of osteotomy.

\section{Introduction}

Oral squamous cell carcinoma (OSCC) with mandibular invasion is associated with a poor prognosis. ${ }^{1}$ Determination of the presence and extent of mandibular invasion in a patient with OSCC is important for ensuring a complete resection with clear margins and for planning mandibular reconstruction. ${ }^{2}$ However, an accurate intraoperative determination of the surgical margin is difficult because of the high mineral content of the involved tissue and the pathological decalcification procedure. Currently, surgeons rely on preoperative clinical examinations and imaging to determine the presence or absence of bone invasion and the required extent of mandibular resection. ${ }^{3}$ To ensure adequate resection, marginal or segmental mandibulectomy are performed after mandibular invasion is confirmed by imaging. ${ }^{4}$ Moreover, to ensure the maximum preservation of tissue and function, the range of mandibular resection should be determined preoperatively. Given the benefits of resection and preservation, the accurate preoperative definition of mandibular invasion by OSCC is crucial for optimizing treatment and preventing local recurrence.

$\mathrm{CT}$ is the most important tool in the evaluation of mandibular invasion. However, CT is limited by the resolution of tools and differences in the detection method. Cone-beam computed tomography (CBCT) is used widely for mandibular examination and more recently has been to facilitate the evaluation of and 
treatment planning for mandibular cancer. Previous studies have demonstrated the high spatial resolution of CBCT, as well as high sensitivity for the estimation of mandibular invasion by cancer However, no studies have investigated the accuracy of this modality for predicting the extent of invasion.

The present study aimed to determine the accuracy of $\mathrm{CBCT}$ for the evaluation of mandibular invasion by OSCC via comparisons with the findings of histopathological analysis, with due consideration of the bone mass shrinkage that occurs during tissue processing. For this study, we used CBCT with a resolution of $0.25 \mathrm{~mm}$, which is much smaller than the 3-mm resolution of conventional 64 -slice spiral CT.

\section{Materials And Methods}

The present study was approved by the Human Ethics Committee of the Medical School of Nanjing University, Nanjing Stomatological Hospital, China. All patients provided written informed consent for participation.

\section{Sample acquisition and pretreatment}

Thirty mandibular specimens were collected immediately after mandibulectomy for OSCC at Nanjing Stomatology Hospital between June 2015 and June 2017. No patient received any form of preoperative radiotherapy or chemotherapy. After collection, the samples were implanted with three gutta-percha (GP) points, which served as guides for evaluations of CBCT images. Three 1-mm holes were drilled into half of the cancellous bone, and a 1-mm GP point was inserted up to the bottom of each hole. Because GP points are flexible and were inserted into the bottoms of the holes, the points remained firmly in place during subsequent processing. These three GP points enabled pathologists and radiologists to focus on the same locations in samples, and were superior to the use of metallic pins. This process is shown in Figure 1.

\section{Image acquisition and assessment}

Following pretreatment, all specimens were scanned using a CBCT device (KaVo 3D eXam, USA) with a field of view of $16 \times 13 \mathrm{~cm}$ and a resolution of $0.25 \mathrm{~mm}$. The eXamVision software package (version 1.6, USA) was used to delineate and measure the tumor borders. First, the plane including the three GP points was assessed, including the negative or positive status of bone invasion and inferior alveolar nerve invasion. Subsequently, an experienced radiologist delineated the border of invasion. The distances between the GP points were also measured to quantify tissue shrinkage.

\section{Histopathological assessment}

The final pathology report including the assessment of bone invasion was used as the gold standard. Following CBCT, specimens were fixed in $4 \%$ formaldehyde for at least 48 hours. Subsequently, they were decalcified in a mixture of formic acid, acetic acid, and hydrochloric acid. During decalcification, any superficial bone that became soft was removed, resulting in a thinner specimen with stable GP points. A 
3-mm-thick bone slice with the three GP points exposed on the surface was also acquired (Figure 1C). Finally, the specimens were sliced into 4- $\mu$ m-thick sections that were stained with hematoxylin-eosin. The GP points dissolved during the staining process, and blank circles served as markers of their original positions.

The stained sections were scanned using a digital slice scanning device (NanoZoomer S60, Japan), and the tumor borders were mapped using NDP.view 2 U12388-21 software (version 2.7, Hamamatsu, Japan). The distances between the GP points were measured twice: when the specimens were embedded in paraffin and after staining with hematoxylin-eosin.

\section{Border fusion}

The histopathological images were merged with the CBCT images using Adobe Photoshop CC software (version 14.0, Adobe, USA) to compare the extent of invasion determined by CBCT with that determined by histopathological examination. The images were magnified to enable matching of the GP points in consideration of the bone shrinkage that occurred during histological processing. However, the difference in shrinkage between the tumor lesion and the adjacent bone had little influence because the samples mainly contained healthy bone mass and the border of the tumor tissue.

\section{Statistical analysis}

The data were subjected to a descriptive statistics analysis and paired t-tests. All statistical analyses were performed using SPSS software (version 23, IBM, USA). A $p$-value of $<0.05$ was considered statistically significant.

\section{Results}

Among the 30 included specimens, 15 (50\%) were obtained from patients who underwent segmental mandibulectomy. Thirteen (87\%) of these patients exhibited mandibular invasion, and the characteristics and pathological stages of these specimens are presented in Table 1. The remaining 15 (50\%) patients had undergone marginal mandibulectomy.

\section{Tissue shrinkage}

The distances between the GP points measured during histological processing were significantly smaller than those measured on the CBCT images, suggesting that bone samples experienced significant shrinkage during tissue processing. Moreover, the distances measured after hematoxylin-eosin staining were larger than those measured after paraffin embedding. The tissue shrinkage ratios determined from histopathological and CBCT measurements are shown in Figure 2A. When all tissue shrinkage ratios during histological processing were considered, the overall ratio was $91.1 \%$ ( $95 \%$ confidence interval: 90.2-92.0\%). 
Overall, $43 \%$ specimens exhibited bone invasion. CBCT correctly predicted the invasion in all specimens, thus yielding sensitivity and specificity rates of $100 \%$. However, tumor invasion was sometimes difficult to identify if other low-density structures such as the inferior alveolar nerve canal were present in the relevant area. Invasion of the inferior alveolar nerve was evident in $46.2 \%$ of specimens exhibiting bone invasion, although the sensitivity and specificity of $\mathrm{CBCT}$ for the prediction of nerve invasion were only $66.7 \%$ and $71.4 \%$, respectively. Table 2 presents the descriptive statistics, positive and negative predictive values, and accuracy of CBCT for the prediction of inferior alveolar nerve invasion by OSCC. A visual observation of the merged images revealed differences in the extent of invasion determined via histopathological examination and CBCT. Figure 2B depicts the largest difference for each specimen. $\mathrm{CBCT}$ tended to underestimate the extent of invasion relative to that determined via histopathological examination, with an average difference of $2.97 \mathrm{~mm}$ according to the largest distance. Figure 3 shows the matched tumor borders delineated by the two methods.

\section{Observation of mandibular invasion}

Mandibular invasion by OSCC can be erosive or infiltrative. ${ }^{5}$ In the present study, the erosive pattern was characterized by a fibrotic interface invaded by numerous lymphocytes between the tumor and bone, with no bone islands within the tumor (Figure 4A). The infiltrative pattern was characterized by several nests of tumor cells along an irregular advancing boundary, with bone islands within the tumor (Figure 4B). Unfortunately, these two types cannot be distinguished by CBCT.

\section{Discussion}

Our research aimed mainly to evaluate the accuracy of $\mathrm{CBCT}$ for assessing the extent of mandibular invasion. We hypothesized that CBCT could help to narrow the area of surgical resection and increase the precision of mandibular resection. Meanwhile, we also studied the accuracy of CBCT for detecting nerve invasion, which we considered an experimental topic of interest. To date, no studies have investigated the accuracy of CBCT for predicting the extent of invasion. However, previous studies have compared invasion lengths and depths determined on orthopantomograms (OPG), bone scans, and CT images with those determined via histopathological examination. ${ }^{6}$ In a previous report, OPG underpredicted the width and depth of invasion by averages of $13 \mathrm{~mm}$ and $2 \mathrm{~mm}$, whereas CT underpredicted the width of invasion by $5 \mathrm{~mm}$ and overpredicted the depth by $3 \mathrm{~mm}$. Our study revealed an average underestimation of $2.97 \mathrm{~mm}$ by CBCT. In other words, CBCT provided a more accurate assessment of the extent of mandibular invasion.

CBCT has a high spatial resolution and reduces the radiation dose to patients; thus, it is useful for the prediction of mandibular invasion. Numerous studies have investigated the utilities of different modalities used to determine mandibular invasion. A systematic review by Rojas and colleagues in 2013 demonstrated that several modalities yielded highly accurate results when detecting mandibular invasion by OSCC, with sensitivity values of $94 \%$ for magnetic resonance imaging, $91 \%$ CBCT, $83 \%$ for CT, and $55 \%$ for panoramic radiography. ${ }^{7}$ Moreover, the authors of that review observed specificity values of $100 \%$ for 
magnetic resonance imaging, CBCT, and CT; $97 \%$ for positron emission tomography/CT; and $91.7 \%$ for panoramic radiography. In 2017, Czerwonka and colleagues compared CBCT with conventional CT and reported that the sensitivity and specificity values for CBCT were $91 \%$ and $60 \%$, respectively, whereas those for CT with bone windows were $86 \%$ and $68 \%$, respectively. ${ }^{8}$ These reports provide a basis for the use of CBCT in evaluations of tumor bone invasion. In our study, CBCT predicted bone invasion with $100 \%$ accuracy, a higher level than those reported in other studies. We suspect that this discrepancy was attributable to our use of in vitro samples, indicating that this method may increase the accuracy of СВСТ. However, the results still prove that СВCT is a fairly accurate tool. In addition, the improved resolution of $\mathrm{CBCT}$ may allow us to make more precise surgical guides.

Notably, we found that CBCT could not predict inferior alveolar nerve invasion at a high level of accuracy. We attribute this finding to the lack of distinction between tumor invasion and the inferior alveolar neural tube in CBCT. However, more studies of CBCT are needed, and the evaluation of additional cases will enable the confirmation and verification of our results.

In our study, the bone specimens exhibited significant linear changes during histopathological examination. During histological processing, tissue shrinkage occurs as a consequence of the fixation and subsequent serial dehydration and rehydration procedures. ${ }^{9}$ Buytaert and colleagues reported a bone volume shrinkage rate of $17 \%$ during tissue processing. ${ }^{10}$ However, our study revealed more details of these changes, including shrinkage and enlargement. Previous reports have described the high significance of OSCC margin discrepancies after resection and specimen processing, as these might influence the adequacy of resection. ${ }^{11,12}$ Therefore, bone shrinkage should be considered in studies involving the sectioning of bone for histopathological examination. Our finding may promote improvements in the accuracy of pathology-based research.

GP points played an important role in our research. The three GP points embedded in the samples not only enabled the pathologist and radiologist to focus on the same locations within samples, but also were utilized as markers to decrease the influence of shrinkage. As GP points are flexible and were inserted into the bottoms of the tissue holes, they could remain firmly in place until the specimen was sectioned. Accordingly, the GP points are superior to markers such as metallic pins, which shift easily during histopathology processing. Thus, GP points may be a very useful tool in imaging research. However, this method still has shortcomings. The pathological examination used 4 - $\mu \mathrm{m}$-thick sections, which were considerably thinner than the GP points. This defect could have led to errors in the merged images. Nevertheless, the differences between different planes that included GP points were very small. Although this technique is prone to error, it also yields substantial improvements.

As mentioned earlier, mandibular invasion by OSCC can be erosive or infiltrative. ${ }^{5,13-16}$ The erosive pattern is characterized by a broad advancing boundary, with a well-defined interface between the tumor and the bone. Osteoclastic bone resorption and fibrosis are typically evident along the advancing boundary and support the absence of bone islands within the tumor mass. In contrast, the infiltrative pattern is characterized by nests and projections of tumor cells along an irregular advancing boundary, 
residual bone islands within the tumor, and haversian system penetration. The presence of features of both patterns suggests a mixed-pattern invasion. Unfortunately, we did not observe distinguishing features related to these invasive types on CBCT images. Therefore, the improvement of preoperative examination techniques remains a huge challenge.

The validation of medical imaging tools is an area of great clinical interest, and highly accurate coregistration between histopathological and radiological images in terms of the tumor boundaries can provide further clarity. The findings of this study suggest that researchers should consider bone shrinkage as a means of improving the accuracy of future bone studies. GP points can be utilized as markers to decrease the influence of shrinkage. Moreover, CBCT is a reliable and highly accurate method for predicting mandibular invasion, but is considerably less accurate in terms of estimating nerve invasion. The calculated underestimation of invasion of $2.97 \mathrm{~mm}$ by CBCT, which was lower than previously reported values, suggests an enormous potential for narrowing the extent of mandibulectomy for mandibular preservation.

\section{Declarations}

Conflict of Interest Statement

The authors declare that they have no conflicts of interest.

\section{References}

1. Guerra MFM, Gias LN, Campo FR, Perez JS: Marginal and segmental mandibulectomy in patients with oral cancer: A statistical analysis of 106 cases. Journal of Oral and Maxillofacial Surgery 61:1289, 2003

2. Shah JP, Gil Z: Current concepts in management of oral cancer-surgery. Oral Oncol 45:394, 2009

3. Pandey M, Rao LP, Shukla M, Sharma V: Mandibular conservation in oral cancer. Surg Oncol 21:109, 2012

4. Costa F, Ferlito A, Politi M, Robiony M, Rinaldo A: Review of segmental and marginal resection of the mandible in patients with oral cancer. Acta Otolaryngol 120:569, 2000

5. Furuta H, Jimi E, Matsuo K, Nakanishi O, Tominaga K, Takahashi T: The cellular and molecular mechanisms of bone invasion by oral squamous cell carcinoma. Oral Dis 17:462, 2011

6. Brown JS, Browne RM, Griffith JF, Phelps PD: A comparison of different imaging modalities and direct inspection after periosteal stripping in predicting the invasion of the mandible by oral squamous cell carcinoma. Br J Oral Maxillofac Surg 32:347, 1994

7. Rojas LA, Rosas CF, Uribe S: Accuracy of imaging methods for detection of bone tissue invasion in patients with oral squamous cell carcinoma. Dentomaxillofac Radiol 42:20120346, 2013

8. Czerwonka L, Bissada E, Goldstein DP, Wood RE, Lam EW, Yu E, Lazinski D, Irish JC: High-resolution cone-beam computed tomography for assessment of bone invasion in oral cancer: Comparison with 
conventional computed tomography. Head Neck 39:2016, 2017

9. Chatterjee S: Artefacts in histopathology. J Oral Maxillofac Pathol 18:S111, 2014

10. Buytaert J, Goyens J, De Greef D, Aerts P, Dirckx J: Volume shrinkage of bone, brain and muscle tissue in sample preparation for micro-CT and light sheet fluorescence microscopy (LSFM). Microsc Microanal 20:1208, 2014

11. Beheiri MG, El-Fol HA, Khalil AM, Kamel MM, Noman SA: Significance of post-resection tissue shrinkage on surgical margins of oral squamous cell carcinoma. Journal of Cranio-Maxillofacial Surgery 43:475, 2015

12. Cheng A, Cox D, Schmidt BL: Oral squamous cell carcinoma margin discrepancy after resection and pathologic processing. J Oral Maxillofac Surg 66:523, 2008

13. Amemiya A, Fukuda H, lizuka T, Shindo M, Totsuka Y, Tei K, Usui Y: Mandibular involvement by squamous cell carcinoma of the lower alveolus: analysis and comparative study of histologic and radiologic features. Head Neck 13:40, 1991

14. Slootweg PJ, Muller H: Mandibular invasion by oral squamous cell carcinoma. J Craniomaxillofac Surg 17:69, 1989

15. Carter RL, Dinsdale EA, Foster CS, Pittam MR: Perineural spread by squamous carcinomas of the head and neck: a morphological study using antiaxonal and antimyelin monoclonal antibodies. $J$ Clin Pathol 36:269, 1983

16. Muller $\mathrm{H}$, Slootweg PJ: Mandibular invasion by oral squamous cell carcinoma. Clinical aspects. J Craniomaxillofac Surg 18:80, 1990

\section{Tables}

Table 1. Characteristics of specimens with mandibular invasion by oral squamous cell carcinoma 


\begin{tabular}{lc}
\hline Category & $\boldsymbol{n}$ \\
\hline Sex & 8 \\
\hline Male & 5 \\
\hline Female & \\
\hline Age (years) & 2 \\
\hline $40-50$ & 4 \\
\hline $50-60$ & 2 \\
\hline $60-70$ & 5 \\
\hline $70-80$ & 5 \\
\hline Site & 8 \\
\hline Lower alveolus & 5 \\
\hline Center of the mandible & 8 \\
\hline N classification & \\
\hline Norosive type & \\
\hline N 1 -2 & \\
\hline Infiltrative & \\
\hline
\end{tabular}

Table 2. Diagnostic accuracy of cone-beam computed tomography for the detection of inferior alveolar nerve invasion by oral squamous cell carcinoma $(n=13)$

\begin{tabular}{|c|c|c|}
\hline TPTNFPFNSensitivity (\%) & Specificity (\%) & Accuracy \\
\hline $\begin{array}{lllll}\text { CBCT4 } & 5 & 2 & 2 & 66.7\end{array}$ & 71.4 & 69.2 \\
\hline
\end{tabular}

true positive; TN, true negative; FP, false positive; FN, false negative; $\mathrm{CBCT}$, cone-beam computed tomography

\section{Figures}



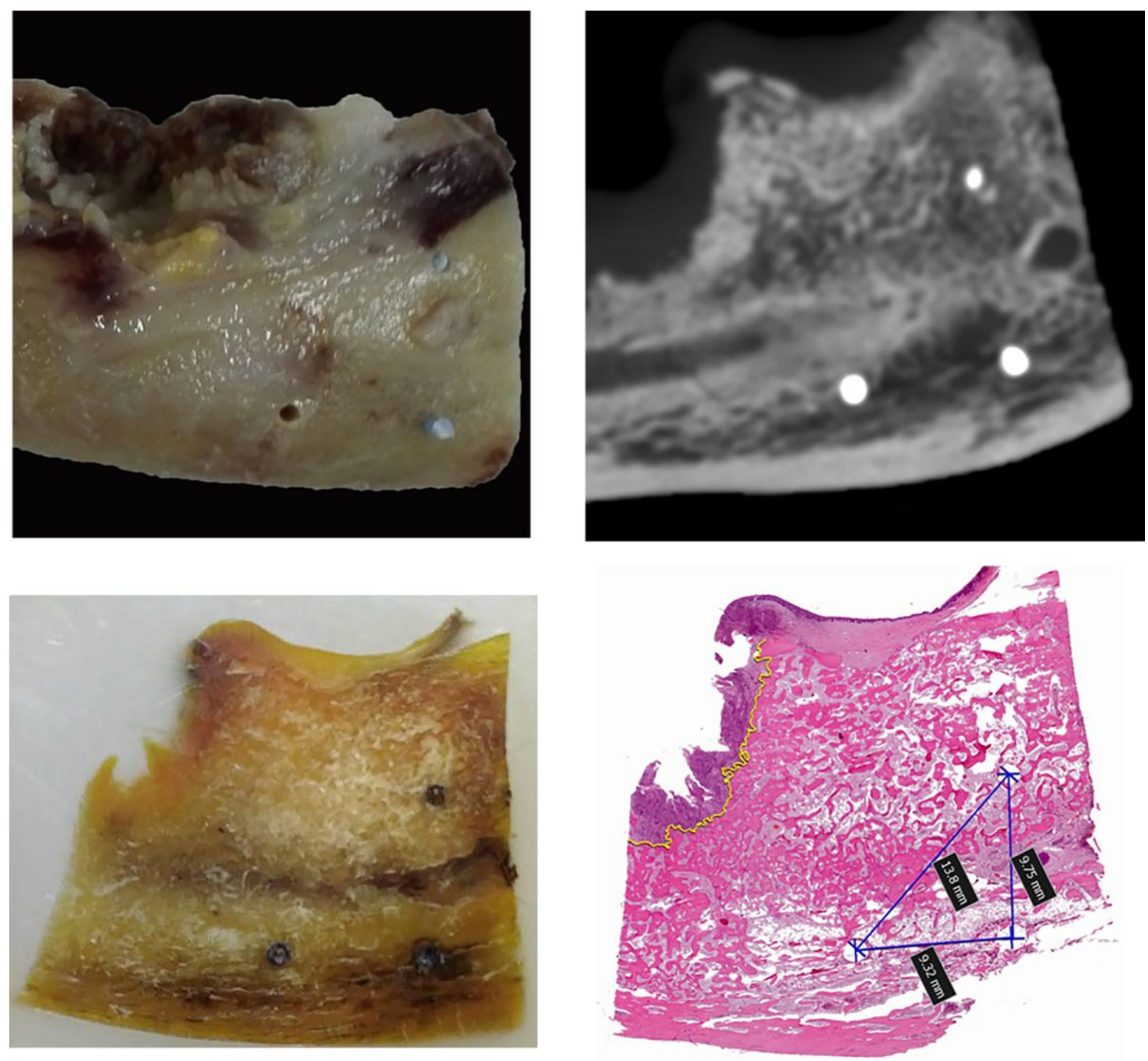

\section{Figure 1}

A. Three gutta-percha points were placed in the samples. B. Gutta-percha points appeared as highlights in a cone-beam computed tomography image. C. Simultaneous surface exposure of three gutta-percha points. D. A section including three gutta-percha points was stained with hematoxylin-eosin; subsequent dissolution of the points yielded three round holes. 

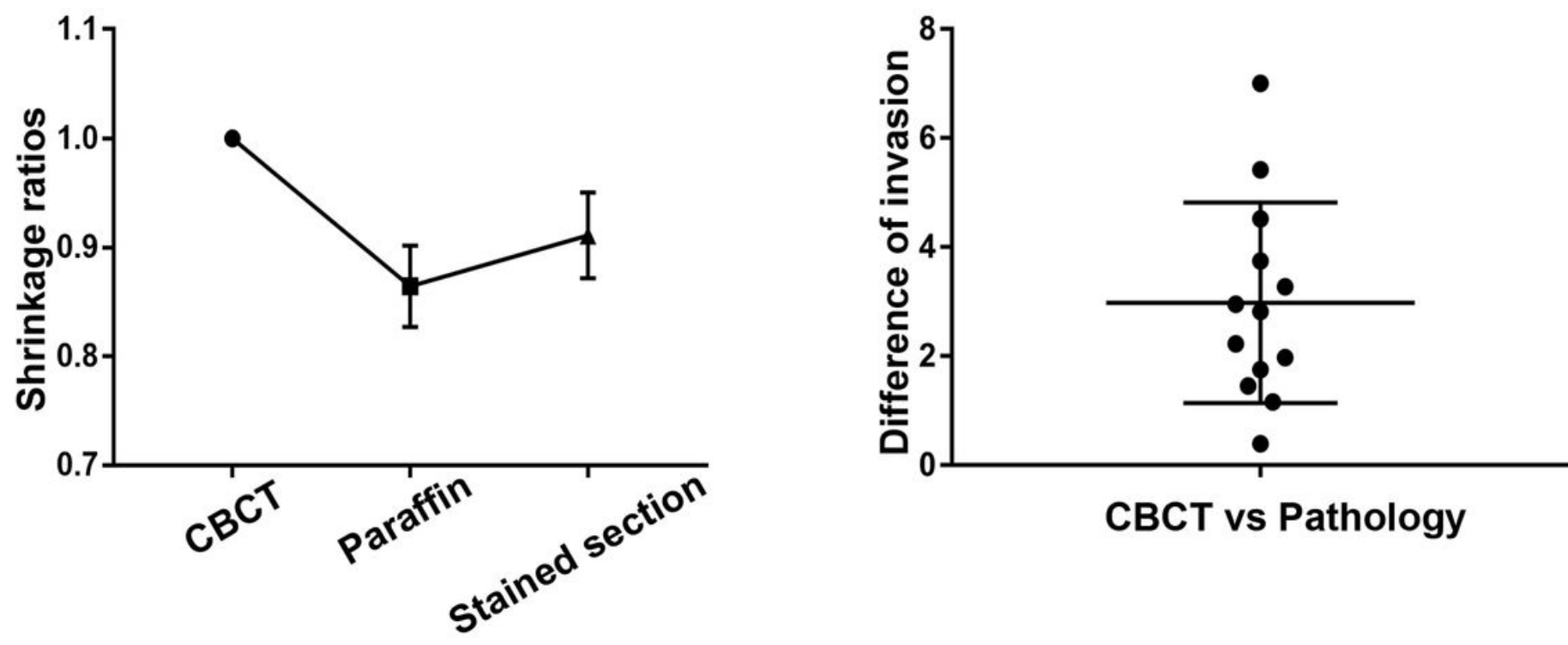

Figure 2

. A. Tissue shrinkage ratios during histological processing. Tissue shrinkage occurred during paraffin embedding and was reversed during subsequent processing. B. The largest difference in the invasive front between CBCT and histopathological images. This largest difference ranged between $0 \neg-7 \mathrm{~mm}$ in each specimen, with an average difference of $2.97 \mathrm{~mm}$. CBCT, cone-beam computed tomography 

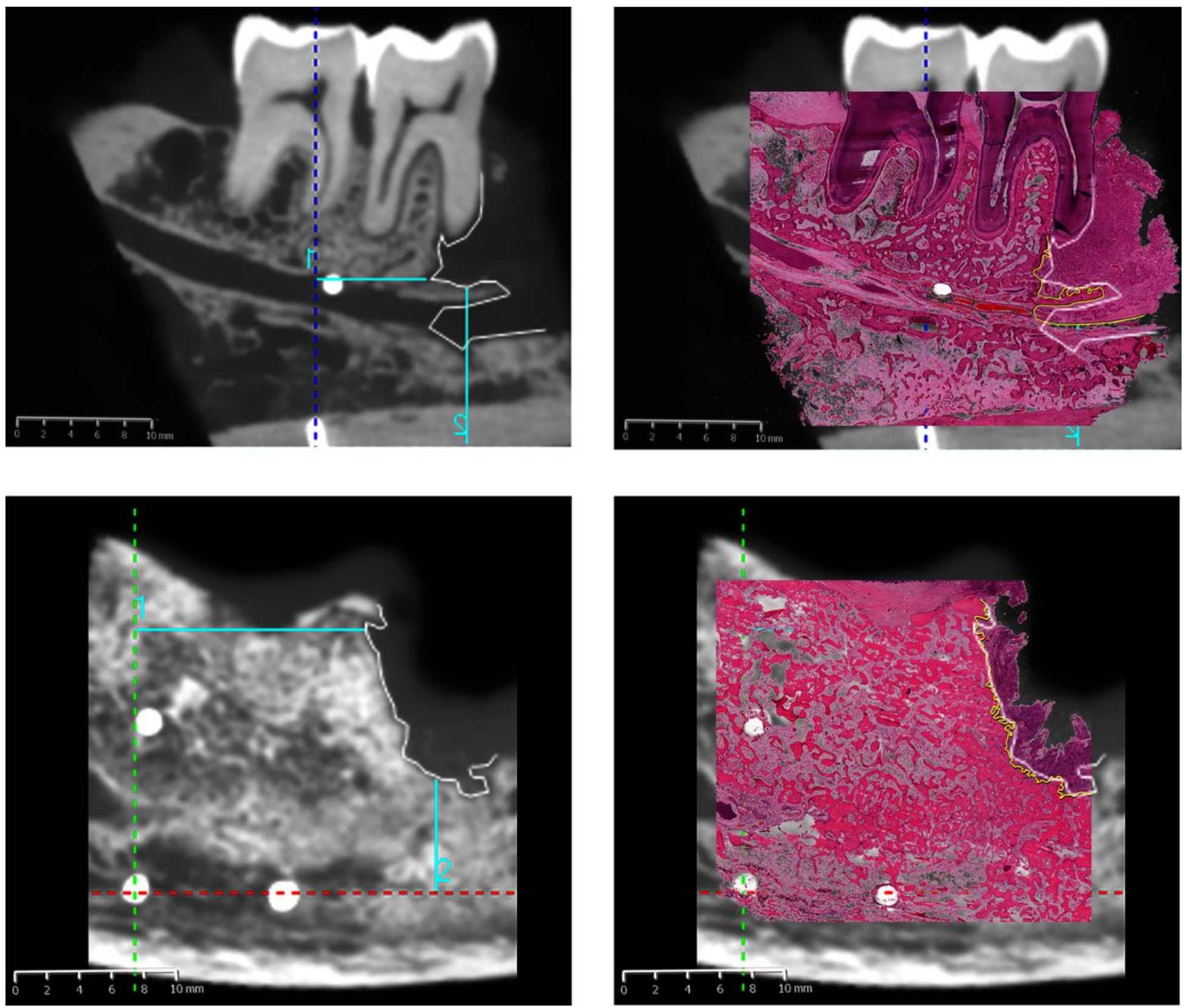

Figure 3

Merged cone-beam computed tomography and histopathological images depicting the tumor borders determined by each modality. A. Mandibular invasion with involvement of the inferior alveolar nerve canal. B. Mandibular invasion distant from the inferior alveolar nerve. The white and yellow lines indicate the borders delineated by cone beam computed tomography and by histopathological examination, respectively. 

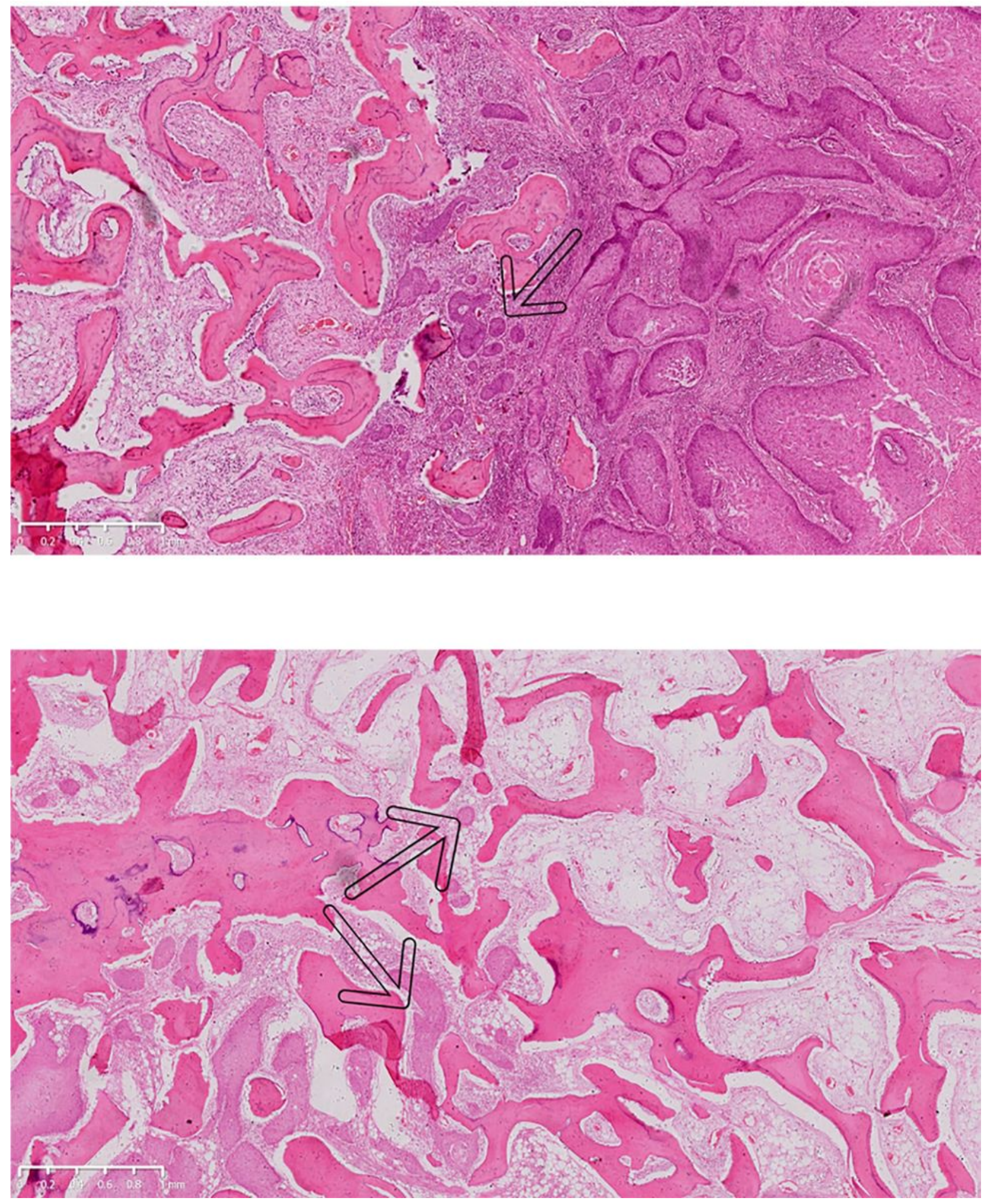

Figure 4

Histopathological examination of mandibular invasion by oral squamous cell carcinoma in hematoxylineosin-stained tissue. A. The erosive pattern of bone invasion is characterized by a fibrotic with many infiltrating lymphocytes $(\times 2.5$ magnification $)$. $B$. The infiltrative pattern is characterized by the presence of several nests and bone islands within the tumor ( $\times 2.5$ magnification). The black arrows indicate tumors. 